\title{
Influence of Smoking Habits on the Prevalence of Dental Caries: A Register-Based Cohort Study
}

\author{
Miguel A. de Araújo Nobre ${ }^{1,2}$ Ana M. Sezinando ${ }^{3}$ \\ ${ }^{1}$ Department of Research, Development and Education, Maló Clinic, \\ Avenida dos Combatentes, Lisboa, Portugal \\ ${ }^{2}$ Clínica Universitária de Estomatologia, Faculdade de Medicina, \\ Universidade de Lisboa, Lisboa, Portugal \\ ${ }^{3}$ Dentistry Department, Private Practice, Porto, Portugal \\ ${ }^{4}$ Department of Dentistry, Maló Clinic Lisbon, Lisboa, Portugal \\ ${ }^{5}$ Centro de Investigação Interdisciplinar Egas Moniz, Egas Moniz \\ University Institute, Caparica, Portugal
}

\author{
Inês C. Fernandes ${ }^{4,5}$ Andreia C. Araújo ${ }^{1}$
}

\begin{abstract}
Address for correspondence Miguel A. de Araújo Nobre, RDH, MSc, PhD, Department of Research and Development, Maló Clinic Lisbon, Avenida dos Combatentes, 43, piso 11, 1600-042 Lisbon, Portugal (e-mail: mignobre@gmail.com,mnobre@maloclinics.com).
\end{abstract}

\author{
Abstract \\ Keywords \\ - dental caries \\ - smoking \\ - socioeconomic \\ factors \\ - risk
}

Objective This study aimed to evaluate the influence of smoking habit on the prevalence of dental caries lesions in a follow-up study.

Materials and Methods A total of 3,675 patients (2,186 females and 1,489 males) with an average age of 51.4 years were included. Outcome measures were the incidence of dental caries defined as incipient noncavitated, microcavitated, or cavitated lesions which had been diagnosed through clinical observation with mouth mirror and probe examination evaluating change of texture, translucency, and color; radiographic examination through bitewing radiographs; or secondary caries through placement of a new restoration during the follow-up of the study.

Statistical Analysis Cumulative survival (time elapsed with absence of dental caries) was estimated through the Kaplan-Meier product limit estimator with comparison of survival curves (log-rank test). A multivariable Cox proportional hazards regression model was used to evaluate the effect of smoking on the incidence of dental caries lesions when controlled to age, gender, systemic status, frequency of dental hygiene appointments, and socioeconomic status. The significance level was set at $5 \%$.

Results Eight hundred sixty-three patients developed caries (23.5\% incidence rate). The cumulative survival estimation was $81.8 \%$ and $48 \%$ survival rate for nonsmokers and smokers, respectively $(p<0.001)$, with an average of 13.5 months between the healthy and diseased state diagnosis. Smokers registered a hazard ratio for dental caries lesions of $1.32(p=0.001)$ when controlled for the other variables of interest.

Conclusion Within the limitations of this study, it was concluded that smoking habit might be a predictor for dental caries. published online July 24, 2021
DOI https://doi.org/

$10.1055 / \mathrm{s}-0041-1729458$ ISSN 1305-7456 (c) 2021. European Journal of Dentistry.

This is an open access article published by Thieme under the terms of the Creative Commons Attribution-NonDerivative-NonCommercial-License, permitting copying and reproduction so long as the original work is given appropriate credit. Contents may not be used for commercial purposes, or adapted, remixed, transformed or built upon. (https://creativecommons.org/licenses/by-nc-nd/4.0/).

Thieme Medical and Scientific Publishers Pvt. Ltd. A-12, 2nd Floor, Sector 2, Noida-201301 UP, India 


\section{Introduction}

Smoking represents one of the major risk factors for lung cancer and cardiovascular disease ${ }^{1}$ with a well-established causal relationship. The relation between smoking and carcinoma of the lung is known since 1950 by Doll et al. ${ }^{2}$ The impact of tobacco on mortality is severe, with 6 million persons dying every year according to the World Health Organization. ${ }^{3}$ Nevertheless, the consumption of tobacco remains high worldwide, with a $21 \%$ rate. ${ }^{4}$

Smoking habits also impact directly the oral cavity, with a wide range of effects extending from teeth/dental fillings staining, to periodontal disease, increased failure rates for dental implants and oral cancer. ${ }^{5}$ According to the International Agency for Research on Cancer, the estimated number of incident cases for lip and oral cavity cancer worldwide in 2012 was 300,000, with 145,000 estimated deaths. ${ }^{6}$

Systemic and oral diseases may share common risk factors. Consequently, a recent review suggested appropriate risk factor management procedures to be adopted in the dental setting ${ }^{7}$ : smoking cessation, reduction of sugar consumption, and weight control were proposed for patients at risk of periodontal disease, caries, diabetes, heart disease, and certain cancers.

Dental caries is the most prevalent disease worldwide, ${ }^{7}$ with a majority of caries lesions being concentrated often in disadvantaged social groups: increased odds for dental caries (21-48\%) were registered in patients with (own or parental) low educational/occupational background and income. ${ }^{8}$ According to a population-based study in Sweden between 1983 and 2003, the relatively unchanged status of tobacco consumption together with a significantly lower frequency of dental visits from smokers compared with nonsmokers reveal a probable trend for an increase in the global burden of dental caries. ${ }^{9}$

The influence of smoking on dental caries is subject of further research, with some authors suggesting an association between an increased risk of dental caries with tobacco smoking. ${ }^{10-12}$ However, the authors of two of these studies (systematic reviews) ${ }^{10,11}$ indicated an overall poor quality of the studies included that prevented validating the association between smoking and dental caries.

The aim of this study was to evaluate the influence of tobacco smoking on the prevalence of dental caries lesions.

\section{Materials and Methods}

This register-based clinical study was written by following the Strengthening the Reporting of Observational Studies in Epidemiology (STROBE) guidelines. ${ }^{13}$ This clinical study was performed in a private clinic in Lisbon, Portugal between July 2012 and December 2014, and it was approved by an independent ethical committee (Ethical Committee for Health, Lisbon, Portugal; authorization no. 005/2012). All procedures in this investigation on the participants were conducted in accordance with the Declaration of Helsinki. All procedures were performed with the adequate and understanding and written consent from all participants included in the study.
The inclusion criteria were patients that presented permanent teeth without active carious lesions at the first diagnostic appointment. There was a total of 3,675 patients included in this study $(2,186$ females and 1,489 males; average age $=51.4$ years; range $=18-97$ years). The clinical examinations were performed by a team trained and calibrated clinicians (with interexaminer reliability results of 0.87 to 0.89 considering the weighted kappa scores in the 3 years of data collection). The patients were part of an epidemiological surveillance study carried to assess the incidence rate of oral diseases. The patient's attendance pattern was characterized by regular check-ups, with patient enrolment in a maintenance program that included dental hygiene and lifestyle instructions (dietary and smoking cessation) and maintenance procedures (scaling, polishing of teeth surfaces, and topical fluoride). The outcomes were recorded at each visit in an anonymized database. For the present study, the information of each patient was retrieved from a register-based database. The information collected from the sample is outlined in - Table 1 and included demographic variables (age and gender), socioeconomic status evaluated from the occupation of each patient according to Goldthorpe classification (socioeconomic status 1: higher managerial, administrative, and professional occupations; socioeconomic status 2: intermediate occupations; socioeconomic status 3: routine and manual occupations), ${ }^{14}$ recall regimen (average frequency of 6 months between dental hygiene appointments), systemic compromise status (absence, $n=2,692$ patients; presence, $n=983$ patients: hepatitis, $n=26$ patients; cardiovascular, $n=636$ patients; thyroid, $n=128$ patients; diabetes, $n=104$ patients; rheumatological, $n=174$ patients; $\mathrm{HIV}+, n=6$ patients; oncological, $n=40$ patients; inflammatory, $n=10$ patients; neurological, $n=13$ patients; autoimmune, $n=5$ patients; renal, $n=3$ patients; genetic, $n=3$ patients; Sjögren syndrome, $n=1$ patient; more than one condition: $n=157$ patients), smoking status (nonsmoker; smoker), and dental caries status (absence or presence).

Outcome measures were the incidence of active dental caries considering noncavitated, microcavitated, or cavitated lesions, ${ }^{15-18}$ which diagnosed by (1) clinical observation with mouth mirror and probe examination (blunt probe, Hu-Friedy, Chicago, Illinois, United States) evaluating change of texture, translucency, and color; (2) radiographic examination through bitewing radiographs (Kodak film bitewing DF42, Eastman Kodak Company, Rochester, New York, United States; Kwik-bite, Kerr, Bioggio, Switzerland); or (3) secondary caries through the placement of a new restoration. Survival was defined as the absence of dental caries during the follow-up of the study.

The onset of dental caries was defined as the time period elapsed between the healthy state and the diagnosis of dental caries during the follow-up of the study.

\section{Statistical Analysis}

The data was analyzed by using SPSS version 17.0 (IBM, Rochester, New York, United States). Descriptive statistics were computed for the variables age, gender, socioeconomic status, systemic compromise status (hepatitis, cardiovascular condition, thyroid condition, diabetes, rheumatologic condition, HIV+, oncological condition, inflammatory condition, 
Table 1 Sample characteristics

\begin{tabular}{|c|c|c|c|}
\hline Variables & $\begin{array}{l}\text { Total } \\
\text { ( } n=3675 \text { patients) }\end{array}$ & $\begin{array}{l}\text { Dental caries } \\
\text { ( } n=670 \text { patients) }\end{array}$ & $\begin{array}{l}\text { Healthy } \\
\text { ( } n=3005 \text { patients) }\end{array}$ \\
\hline Age mean (standard deviation) & $51.4(14.8) y$ & $50.3(15.3) y$ & $51.8(14.7) y$ \\
\hline \multicolumn{4}{|l|}{ Gender } \\
\hline Female & $2,186(100 \%)$ & $505(23.1 \%)$ & $1,681(76.9 \%)$ \\
\hline Male & $1,489(100 \%)$ & $358(24 \%)$ & $1,131(76 \%)$ \\
\hline \multicolumn{4}{|l|}{ Smoking habits } \\
\hline Nonsmoker & $3,005(100 \%)$ & $675(22.5 \%)$ & $2,330(77.5 \%)$ \\
\hline Smoker & $670(100 \%)$ & $188(28.1 \%)$ & $482(71.9 \%)$ \\
\hline \multicolumn{4}{|l|}{ Systemic compromise } \\
\hline Healthy & $2,692(100 \%)$ & $621(23.1 \%)$ & $2,071(76.9 \%)$ \\
\hline Systemic compromised & $983(100 \%)$ & $242(24.6 \%)$ & 741 (75.4\%) \\
\hline $\begin{array}{l}\text { Frequency of dental hygiene appointments: } \\
\text { mean (standard deviation) }\end{array}$ & $6.3(2.6) \mathrm{mo}$ & $6.7(3.4) \mathrm{mo}$ & $6.2(2.3) \mathrm{mo}$ \\
\hline \multicolumn{4}{|l|}{ Socioeconomic status } \\
\hline Status 1 & $2,261(100 \%)$ & $535(23.7 \%)$ & $1,726(76.3 \%)$ \\
\hline Status 2 & $660(100 \%)$ & $152(23 \%)$ & $508(77 \%)$ \\
\hline Status 3 & $395(100 \%)$ & $95(24.1 \%)$ & 300 (75.9\%) \\
\hline
\end{tabular}

Note: Socioeconomic status classification according to Goldthorpe ${ }^{14}$ :

Status 1: Higher managerial, administrative, and professional occupations.

Status 2: Intermediate occupations.

Status 3: Routine and manual occupations.

neurologic condition, autoimmune condition, renal condition, and Sjögren syndrome), smoking status, dental caries status, and onset of dental caries. Cumulative survival was estimated through the Kaplan-Meier product limit estimator and by using the patient as the unit of statistical analysis (first incidence of a dental caries lesion was considered as event). Differences between survival curves were determined by using the log-rank test. The multivariable Cox proportional hazards regression model was used to evaluate the effect of smoking on the incidence of dental caries lesions controlled for the presence of other covariates/confounders. The covariates inserted in the model were age, gender, smoking status, systemic compromise status, frequency of dental hygiene appointments, and socioeconomic status. Regression coefficients (hazard ratios [HR]) were estimated with corresponding 95\% confidence intervals (95\% CI). The survival function was tested for prediction by using the area under the curve statistic (AUC) and illustrated by the receiver operating characteristics curve (ROC curve). The level of significance was set at $5 \%$.

\section{Results}

From 4,179 patients eligible for follow-up, 504 (12\%) were lost to follow-up and excluded from analysis, with analysis performed on the data of the remaining 3,672 patients. During the follow-up of the study, 2,812 patients had no incidence of dental caries while 863 patients developed dental caries (incidence rate of 23.5\%). The average follow-up for each patient was 2 years (range $=1-2.5$ years). The average time of onset for dental caries was 13.5 months $95 \%$ CI (13.0-13.9).
The distributions of survival rate estimates (KaplanMeier) were the following: estimated cumulative survival rates for females and males were 59.5 and $59.8 \%$, respectively, with a nonsignificant difference between both groups ( $p=$ 0.433 ); estimated cumulative survival rates for nonsmokers and smokers was 81.8 and $48.0 \%$, respectively, with a significant difference between both groups ( $p<0.001$; - Fig. 1); estimated cumulative survival rates for healthy and systemic compromised patients was 59.1 and $60.7 \%$, respectively, with a nonsignificant difference between both groups ( $p=0.504$ ); and estimated cumulative survival rates for socioeconomic

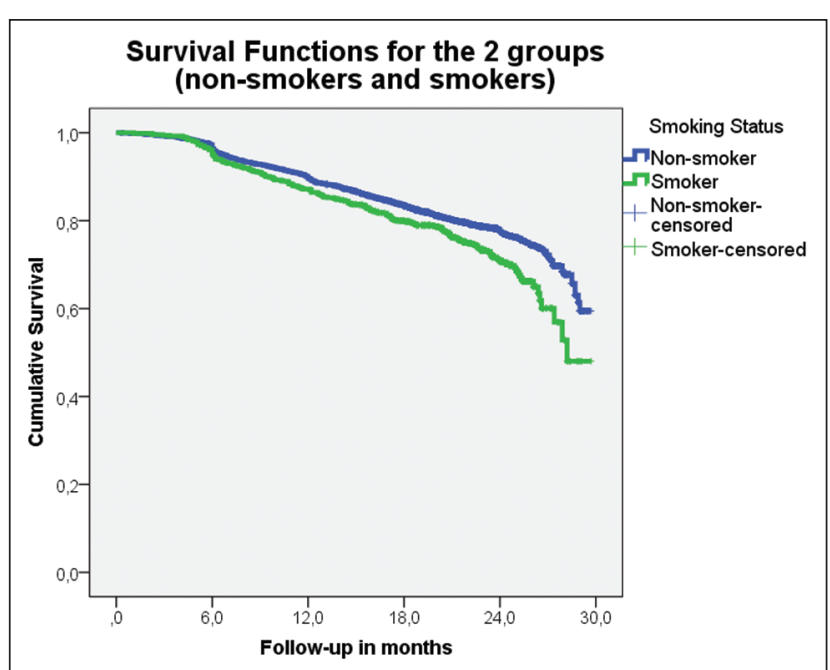

Fig. 1 Kaplan-Meier survival curves for dental caries lesions in nonsmoker and smoker patients; $p<0.001$, hazard ratio $=1.316,95 \%$ confidence interval $=1.114-1.555$. 
status 1,2 , and 3 were $58.5,53.3$, and $70.8 \%$, respectively, with a nonsignificant difference between both groups ( $p=0.943$ ).

The multivariable model identified smoking habits $(H R=1.32)$ as a predictor for dental caries lesions and age as a protective effect $(\mathrm{HR}=0.99)$ when controlled for gender, systemic compromised status, frequency of dental hygiene appointments, and socioeconomic status (- Table 2). The survival function retrieved from the model by using the six predictors registered an AUC of 0.835 and $95 \% \mathrm{CI}$ (0.815-0.855; - Fig. 2).

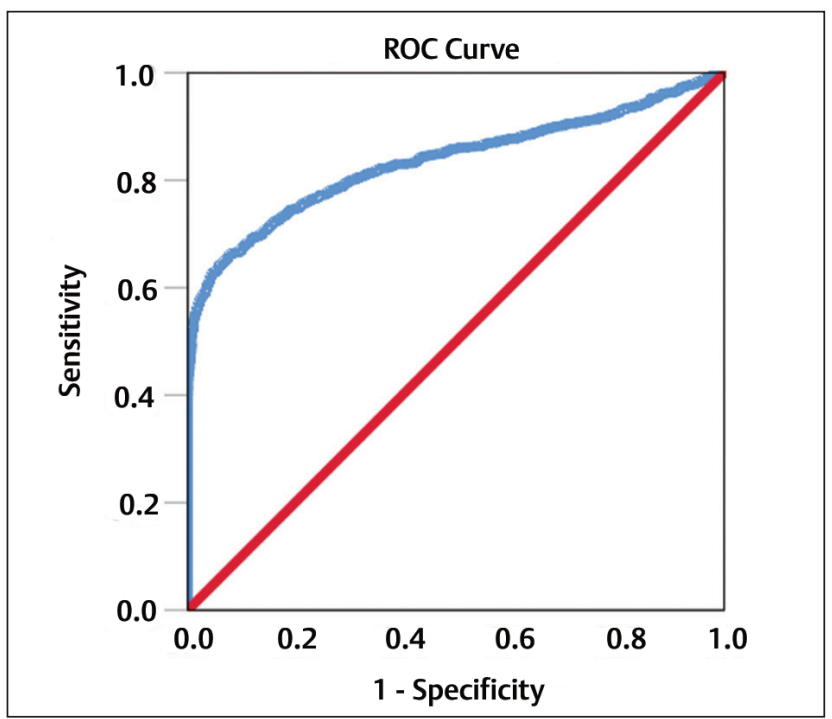

Fig. 2 Receiver operating characteristic curve illustrating the performance of the regression model in prediction of dental caries lesions. The model accuracy according to the C-statistic ( $95 \%$ confidence intervals) was area under the curve $=0.835(0.815-0.855)$.

\section{Discussion}

This study registered a significant association between smoking habits and dental caries prevalence, with a $32 \%$ increase in the hazard ratio for developing dental caries for smokers compared with nonsmokers. This result is in line with the literature ${ }^{10,11,19}$ using a register-based study design and considering the control for other variables of interest. A systematic review ${ }^{10}$ evaluated the effect of tobacco smoking on dental caries in adult smokers and disclosed a significant association between the exposure to smoking and an increased risk of dental caries. Bernabé et $\mathrm{al}^{12}$ in a study that included 955 adult patients, assessed the impact of daily smoking on the 4-year net increment in the numbers of decayed, filled, and missing teeth also reported an increased incidence of dental caries in smokers. In the referred study, the authors concluded that smoking was independently related to caries development with a $70 \%$ increment on the incidence rate ratio of decayed teeth for smokers when compared with nonsmokers.

Smoking might potentially influence the incidence of dental caries through the negative impact on the patients' saliva. The saliva properties of smokers include a higher count of bacteria (Streptococcus mutans) ${ }^{18}$ and a decreased buffering effect that may increase the patient's susceptibility to dental caries, ${ }^{18,20}$ accounting for a $25 \%$ of the variability of caries risk. ${ }^{20}$ Furthermore, smoking was previously associated with lower salivary secretory immunoglobulin A ( $\operatorname{IgA})$ concentrations. $^{21}$ The specificity of $\operatorname{IgA}$ action represents the main specific defense mechanism in the oral cavity. ${ }^{22}$ In conjunction with other antimicrobial substances, the IgA action limits microbial adherence to epithelial and tooth surfaces helping to maintain the oral cavity stable. ${ }^{23-25}$ Smoking

Table 2 Multivariate Cox regression model for risk factors for dental caries lesions (total $n=3,675$ patients)

\begin{tabular}{|l|l|l|l|}
\hline Factor & Hazard ratio & 95\% confidence interval & $p$-Value \\
\hline Age & 0.991 & $(0.986-0.996)$ & 0.001 \\
\hline Gender & & & \\
\hline Female & 1.0 & $(0.897-1.196)$ & 0.635 \\
\hline Male & 1.036 & & \\
\hline Smoking habits & & & 0.001 \\
\hline Nonsmoker & 1.0 & $(1.114-1.554)$ & \\
\hline Smoker & 1.316 & & \\
\hline Systemic compromise & & & 0.057 \\
\hline Healthy & 1.0 & $(0.995-1.409)$ & 0.879 \\
\hline Systemic compromised & 1.184 & $(0.975-1.030)$ & 0.735 \\
\hline Frequency of dental hygiene appointments & 1.002 & & \\
\hline Socioeconomic status & & & $(0.820-1.181)$ \\
\hline Status 1 & 1.0 & $(0.868-1.356)$ & 0.864 \\
\hline Status 2 & 0.984 & 1.085 & 0.473 \\
\hline Status 3 & & \\
\hline
\end{tabular}

Note: Socioeconomic status classification according to Goldthorpe ${ }^{14}$ :

Status 1: Higher managerial-administrative and professional occupations.

Status 2: Intermediate occupations.

Status 3: Routine and manual occupations. 
was also associated to lower salivary cystatin activity and cystatin $\mathrm{C}^{26}$ that contributes to oral health by inhibiting certain proteolytic enzymes suggested to have a contributory effect on caries lesion formation when in interaction with acid demineralization. ${ }^{27}$

Age had a protective effect in our study $(\mathrm{HR}=0.99)$, a result that was also registered in a previous study ${ }^{12}$ and that can be explained by the exposure time. With increased age, there is a higher probability of patients with missing or filled teeth and therefore a lower conditional probability of incident dental caries lesions. The variable socioeconomic status did not yield statistical significance, opposing the results from other study. ${ }^{8}$ Schwendicke et $\mathrm{a}^{8}$ registered a $29 \%$ increase in the odds ratio of caries experience for low income compared with high income in their meta-analysis, despite grading the included studies between low and very low evidence levels due to risk of bias. A probable reason for the different result may reside in the study setting, with the present study performed on a private practice where patients attended a recall regimen in comparison to the Schwendicke et al meta-analysis, ${ }^{8}$ where population-based studies were included. Nonetheless, the short-term development of dental caries had a similar distribution between the three socioeconomic classes, which accounts for the importance of patient clinical monitoring in preventing the incidence of dental caries lesions.

The multivariable model had an accuracy of 0.835 according to the AUC statistic. This result is considered excellent when evaluating the performance of prediction models. ${ }^{28}$ The high accuracy translates into a high proportion of true positives and true negatives among the total number of patients when predicting the probability of experiencing dental caries. This model may be valid for screening patients with high risk of developing dental caries lesions and may be used as an aid in the decision process for implementing more strict methodologies of dental caries prevention and smoking cessation.

Dental caries lesions consist in a dynamic process resulting from an imbalance between demineralization and remineralization of the dental surface. ${ }^{29}$ The time of onset for dental caries registered in the present study was 13.5 months. According to the authors' knowledge, this is the first time such statistic has been computed. Despite the methodology implemented in patients' data collection and monitoring system, this statistic data should be interpreted with caution as it was dependent on recall appointments. It needs to be further refined to retrieve more precise measurements due to the possibility of an earlier diagnosis.

The clinical implications of the time of onset for dental caries is three partied. First, concerning the recall regimen, the results of the present study suggest reducing the recall periods for patients who smoke due to the higher probability of developing dental caries registered in the study; second, a reduced recall regimen would potentially increase the frequency of diagnostic measures; and third, it would enable monitoring the patient adherence to dietary, smoking and oral hygiene habits.

The limitations of this study include the short-term follow-up that could impact the prevalence rate of carious lesions and the lack of control for dietary and oral hygiene habits (considering it was a register-based study with no intervention on patients) that could have provided a more precise estimation of the effect of smoking on dental caries. Nevertheless, the study sample consisted in patients enrolled in a maintenance program, part of an epidemiological surveillance study, where all patients received information on good dietary and oral hygiene habits and smoking cessation as per protocol on the dental hygiene appointments. Furthermore, the results were controlled for the recall regimen (frequency of dental hygiene appointments). It was previously stated in the literature, that smoking may be associated with decreased oral hygiene habits with increased plaque accumulation ${ }^{30-32}$ either related to decreased toothbrushing and flossing frequency ${ }^{32}$ or due to elevated salivary lipid levels. ${ }^{33}$ Further investigations should focus on controlled longer follow-up studies to refine the estimates of both the association of smoking and dental caries and the time of onset for dental caries lesions.

In conclusion and considering the limitations of this study, smoking habits might be a predictor for dental caries while age had a protective effect when adjusted for gender, systemic compromise, socioeconomic status, and frequency of dental hygiene appointments.

\section{Funding \\ None. \\ Conflict of Interest \\ None declared.}

\section{References}

1 Pope CA II, Burnett RT, Turner MC, et al. Lung cancer and cardiovascular disease mortality associated with ambient air pollution and cigarette smoke: shape of the exposure-response relationships. Environ Health Perspect 2011;119(11):1616-1621

2 Doll R, Hill AB. Smoking and carcinoma of the lung; preliminary report. BMJ 1950;2(4682):739-748

3 World Health Organization. Health topics tobacco. World Health Organization Press Office; 2020. Available at: https://www. who.int/health-topics/tobacco\#tab=tab_1. Accessed November 24, 2020

4 World Health Organization. Tobacco control monitor. World Health Organization Press Office; 2020. Available at: https://www.who.int/data/gho/data/themes/topics/topic-details/ GHO/gho-tobacco-control-monitor. Accessed November 24, 2020

5 Reibel J. Tobacco and oral diseases. Update on the evidence, with recommendations. Med Princ Pract 2003;12(Suppl 1): 22-32

6 Ferlay J, Soerjomataram I, Ervik M, et al. GLOBOCAN 2012 v1.0, Cancer incidence and mortality worldwide: IARC CancerBase 2013;11. Lyon, France: International Agency for Research on Cancer. Available at: http://globocan.iarc.fr. Accessed June 20, 2018

7 Genco RJ, Genco FD. Common risk factors in the management of periodontal and associated systemic diseases: the dental setting and interprofessional collaboration. J Evid Based Dent Pract 2014;14(Suppl):4-16

8 Schwendicke F, Dörfer CE, Schlattmann P, Foster Page L, Thomson WM, Paris S. Socioeconomic inequality and caries: a systematic review and meta-analysis. J Dent Res 2015;94(1):10-18 
9 Hellqvist L, Rolandsson M, Birkhed D, Hugoson A. Tobacco use in relation to socioeconomic factors and dental care habits among Swedish individuals 15-70 years of age, 1983-2003. Int J Dent Hyg 2009;7(1):62-70

10 Benedetti G, Campus G, Strohmenger L, Lingström P. Tobacco and dental caries: a systematic review. Acta Odontol Scand 2013;71(3-4):363-371

11 Vellappally S, Fiala Z, Smejkalová J, Jacob V, Shriharsha P. Influence of tobacco use in dental caries development. Cent Eur J Public Health 2007;15(3):116-121

12 Bernabé E, Delgado-Angulo EK, Vehkalahti MM, Aromaa A, Suominen AL. Daily smoking and 4-year caries increment in Finnish adults. Community Dent Oral Epidemiol 2014;42(5):428-434

13 von Elm E, Altman DG, Egger M, Pocock SJ, Gøtzsche PC, Vandenbroucke JP; STROBE Initiative. The Strengthening the Reporting of Observational Studies in Epidemiology (STROBE) statement: guidelines for reporting observational studies. Epidemiology 2007;18(6):800-804

14 Goldthorpe JH, On Sociology. 2nd ed. Stanford: Stanford University Press; 2007 91-117

15 Thylstrup A, Bruun C, Holmen L. In vivo caries models-mechanisms for caries initiation and arrestment. Adv Dent Res 1994;8(2):144-157

16 Nyvad B, Machiulskiene V, Baelum V. Construct and predictive validity of clinical caries diagnostic criteria assessing lesion activity. J Dent Res 2003;82(2):117-122

17 Ekstrand KR, Martignon S, Ricketts DJ, Qvist V. Detection and activity assessment of primary coronal caries lesions: a methodologic study. Oper Dent 2007;32(3):225-235

18 Cogulu D, Sabah E, Kutukculer N, Ozkinay F. Evaluation of the relationship between caries indices and salivary secretory IgA, salivary $\mathrm{pH}$, buffering capacity and flow rate in children with Down's syndrome. Arch Oral Biol 2006;51(1):23-28

19 Ashraf Nazir M, Almas K. Awareness about the effects of tobacco consumption on oral health and the possibility of smoking behavior among male Saudi schoolchildren. Eur J Dent 2017;11(1):29-35

20 Voelker MA, Simmer-Beck M, Cole M, Keeven E, Tira D. Preliminary findings on the correlation of saliva $\mathrm{pH}$, buffering capacity, flow, Consistency and Streptococcus mutans in relation to cigarette smoking. J Dent Hyg 2013;87(1):30-37
21 Golpasand Hagh L, Zakavi F, Ansarifar S, Ghasemzadeh O, Solgi G. Association of dental caries and salivary slgA with tobacco smoking. Aust Dent J 2013;58(2):219-223

22 Hamid H, Adanir N, Asiri FYI, Abid K, Zafar MS, Khurshid Z. Salivary IgA as a useful biomarker for dental caries in Down's syndrome patients: a systematic review and meta-analysis. Eur J Dent 2020;14(4):665-671

23 Benderli Y, Erdilek D, Koray F, Telci A, Turan N. The relation between salivary IgA and caries in renal transplant patients. Oral Surg Oral Med Oral Pathol Oral Radiol Endod 2000;89(5):588-593

24 Jalil RA, Ashley FP, Wilson RF, Wagaiyu EG. Concentrations of thiocyanate, hypothiocyanite, 'free' and 'total' lysozyme, lactoferrin and secretory IgA in resting and stimulated whole saliva of children aged 12-14 years and the relationship with plaque accumulation and gingivitis. J Periodontal Res 1993;28(2):130-136

25 Russell MW, Hajishengallis G, Childers NK, Michalek SM. Secretory immunity in defense against cariogenic mutans streptococci. Caries Res 1999;33(1):4-15

26 Lie MA, Loos BG, Henskens YM, et al. Salivary cystatin activity and cystatin $C$ in natural and experimental gingivitis in smokers and non-smokers. J Clin Periodontol 2001;28(10):979-984

27 Clarkson BH, Hall DL, Heilman JR, Wefel JS. Effect of proteolytic enzymes on caries lesion formation in vitro. J Oral Pathol 1986;15(8):423-429

28 Hosmer DW, Lemeshow S, Applied Logistic Regression. 2nd ed. New York: John Wiley \& Sons; 2000 160-163

29 Featherstone JD. The continuum of dental caries-evidence for a dynamic disease process. J Dent Res 2004;83 Spec No C:C39-C42

30 Arowojolu MO, Fawole OI, Dosumu EB, Opeodu OI. A comparative study of the oral hygiene status of smokers and non-smokers in Ibadan, Oyo state. Niger Med J 2013;54(4):240-243

31 Al-Habashneh R, Al-Omari MA, Taani DQ. Smoking and caries experience in subjects with various form of periodontal diseases from a teaching hospital clinic. Int J Dent Hyg 2009;7(1):55-61

32 Broadbent JM, Thomson WM, Boyens JV, Poulton R. Dental plaque and oral health during the first 32 years of life. J Am Dent Assoc 2011;142(4):415-426

33 Palmerini CA, Saccardi C, Ferracci F, Arienti S. Lipid patterns in the saliva of smoking young adults. Hum Exp Toxicol 2011;30(10):1482-1488 